\title{
Metastatic leiomyosarcoma of the excluded stomach: a case report
}

\author{
Nataliê Almeida Silva Lister Arruda Modesto dos Santos ${ }^{1,}$ Vitorino Modesto dos Santos²* \\ ${ }^{1}$ Department of General Surgery, Advanced Program of Oncosurgery and Videolaparoscopy, Public Workers State Hospital Francisco Morato de Oliveira, São Paulo- \\ SP, Brazil. \\ ${ }^{2}$ Department of Internal Medicine, Armed Forces Hospital and Catholic University, Brasília-DF, Brazil.
}

To Cite

Silva NA, Santos LAM, Santos VM. Metastatic leiomyosarcoma of the excluded stomach: a case report. J Gastric Surg 2020; 2(3): 98-101

\section{Publication history}

Received: August 7, 2020

Accepted: August 29, 2020

Article in press: August 30, 2020

Published online: August 31, 2020

\section{*Correspondence to}

Prof. Dr. Vitorino Modesto dos Santos, MD, $\mathrm{PhD}$

Armed Forces Hospital

Estrada do Contorno do Bosque s/n, Cruzeiro Novo

70658-900, Brasília-DF, Brazil. vitorinomodesto@gmail.com

Telephone: + 55-61 39662103

\section{ABSTRACT \\ Background:}

Leiomyosarcoma (LMS) represents about $1 \%$ of primary malignancies of the stomach, usually evolves with hepatic implants in 2-thirds of cases, and the outcome is frequently poor. With an insidious course, late diagnosis and misdiagnosis with other gastric neoplasia occur. Immunohistochemical evaluations are mandatory to confirm the diagnostic hypothesis. Surgical resection has been the more effective treatment of gastric LMS; however, recurrences after resections and distant metastases may develop in up to $50 \%$ of the patients. Doxorubicin, gemcitabine, and docetaxel are therapeutic options, with variable responses.

\section{Case presentation:}

The 52-year-old male herein described with a diagnosis of LMS in the gastric pouch and liver metastasis underwent a Roux-en-Y bypass to treat morbid obesity more than a decade ago. Persistent abdominal pain was a unique symptom, and he had liver metastasis at diagnosis. The initial hypothesis was a metastatic gastrointestinal stromal tumor (GIST) of the excluded stomach and the patient underwent a schedule with imatinib without significant response. After a complete revision of the anatomopathological findings, the patient underwent a new biopsy of the gastric mass, and the immunohistochemical data were consistent with LMS. Then doxorubicin replaced imatinib, later changed by gemcitabine associated with docetaxel. As last control found lesions in the central nervous system, he is under radiotherapy sessions.

\section{Conclusion:}

The diagnosis of gastric LMS often occurs at late stages because of the insidious clinical course. The rate of liver metastasis at diagnosis is high. Besides, the relatively poor response to the alternative management for non-surgical stages of the disease yields severe outcomes.

\section{Keywords:}

Leiomyosarcoma, liver metastasis, Roux-en-Y gastric bypass surgery. 


\section{Background:}

Leiomyosarcoma (LMS) represents about $1 \%$ of primary malignancies of the stomach, evolves with hepatic implants in 2-thirds of cases, and the outcome is often poor [1-7]. Immunosuppression, Epstein-Barr virus, chemicals, and radiation can be related factors $[2,6]$. Spindle cells of the muscularis propria or muscularis mucosa present high proliferation rates and positivity for desmin, a-muscle-specific actin, and vimentin $[2,3,6]$. Although immunohistochemical findings can avoid pitfalls with other gastrointestinal tumors, the diagnosis often occurs at late stages because of the insidious clinical course [2-7]. LMS predominantly affects female adults (5:3) and the sixth decade of life, pain occurs in near 75\% of patients and the control is very difficult in most of them $[1,2]$. Bleeding, weight loss, and gastroesophageal reflux are other frequent symptoms [2-4]. Whenever possible, surgical resection is the effective treatment; otherwise, palliative care and repeated hepatectomy for metastasis resection may prolong the survival rate in some cases [1-5]. Recurrences after resections and distant metastases may develop in up to $50 \%$ of patients [5]. Anthracycline-based chemotherapy, ifosfamide, gemcitabine, docetaxel, trabectedin, and pazopanib constitute additional therapeutic options, with variable response rates [6,7]. Because of the exceeding rarity, case reports can increase the understanding of gastric LMS.

\section{Case Report}

A 53-year-old Caucasian male who underwent a Rouxen-Y gastric bypass (RYGB) in 2005 due to grade III obesity, and laparoscopic cholecystectomy in 2006, was admitted in 2018 claiming of abdominal pain started seven days before. He denied weight loss, nausea, vomiting, and digestive hemorrhage; and reported being a former smoker (five packs/year). There was no antecedent of Epstein-Barr virus infection, Wilms tumor, or immunosuppression. On physical examination, his BMI was $39.20 \mathrm{~kg} / \mathrm{m}^{2}$, with normal vital signs and unremarkable abdomen evaluation. The results of routine laboratory tests were normal. The computed tomography (CT) of the abdomen (Figure 1) showed a hypo vascular abdominal mass with cystic and necrotic degeneration, occupying the epigastrium and the left hypochondrium, with no cleavage plane with the border of the left hepatic lobe, and apparent continuity with the upper body wall of the excluded stomach (measuring $17.0 \mathrm{~cm} \times 10.3 \mathrm{~cm} \times 8.6 \mathrm{~cm}$ ). The diagnostic suspicion was of primary neoplastic involvement, in addition to metastatic liver nodules. The CT of the chest showed a lytic lesion in the fourth left costal arch and solid nodules up to $1.0 \mathrm{~cm}$ distributed in both lungs, suggestive of metastases. Evaluation by upper digestive endoscopy revealed gastric bypass without changes. The colonoscopy procedure detected a rectal polypoid lesion and its resection yielded the diagnosis of leiomyoma. The tumor markers were within the normal range (carcinoembryonic antigen: 0.9, CA 125: 15.6, CA 19-9: <2, and alpha-fetoprotein: 2.6). The biopsy of liver lesions revealed a poorly differentiated fusocellular neoplasia, with immunohistochemistry study indicating a high-grade mesenchymal malignancy with positive Ki-67 and vimentin. Three revisions of the slides did not demonstrate the immunoexpression of CD117.

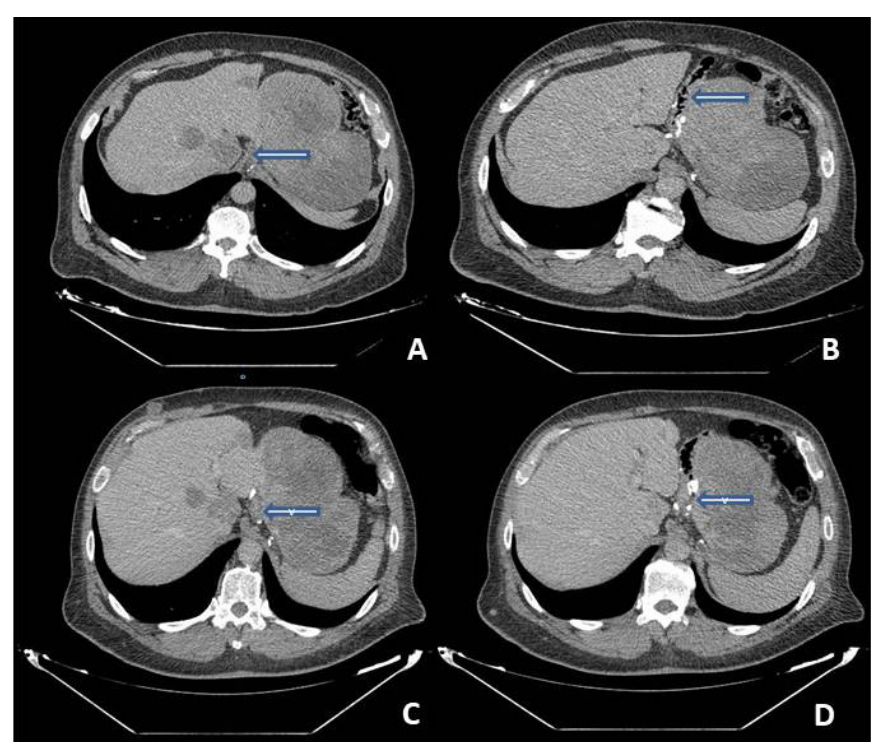

Figure 1: Abdominal CT scan (A and B, 2018) showing a hypovascular cystic-necrotic mass (arrows) in the excluded stomach (gastric pouch), and hepatic nodules; and (C and D, 2019) the persistence of both the hepatic nodules and the mass (arrows) with no apparent cleavage with the gastric pouch.

Considering the strong hypothesis of a metastatic gastrointestinal stromal tumor (GIST) from the excluded stomach, the patient initially underwent a schedule with imatinib (400 mg/day) from July 2018 to June 2019. Although the disease seemed stable with this therapy, the patient underwent another biopsy of the mass in the excluded stomach guided by ultrasound. The immunohistochemical panel showed vimentin V9 and actin 1A4 positive, while CD117 (c-kit), CD34, and S100 protein were negative. These data confirmed the diagnosis of leiomyosarcoma in an excluded stomach. Then, the chemotherapy changed by liposomal doxorubicin $\left(75 \mathrm{mg} / \mathrm{m}^{2}\right)$ from July till October 2019 . Since December he is utilizing docetaxel in association with gemcitabine. The latest control imaging revealed the stability of the hepatic implants, bone lesions, and pulmonary nodules; however, the disease progressed affecting the central nervous system. Currently, the patient is clinically stable under follow-up care by the service of radiotherapy.

\section{Discussion}

The male patient herein described underwent bariatric surgery for the management of his obesity. The RYGB procedure resulted in a reduction of his gastric volume by defining a small proximal gastric pouch without the remaining stomach [8]. This postoperative anatomy change corresponds to the called "excluded stomach" from the gastrointestinal tract. And the persistent alkaline effect of duodenal bile reflux has been related to the gastric cancer risk [8]. Excluded stomachs can harbor a great number of bacteria and fungi, with a variable degree of gastritis, atrophy, and intestinal metaplasia which may predispose to gastric malignancy [8]. An additional concern might be the finding of 5-ciprinol sulphate, a toxic bile alcohol present in certain dysfunctional conditions, including inflammation 
and hepatic carcinogenesis [8]. Notwithstanding, with respect specifically to gastrointestinal LMS, the known predisposing factors include a history of Wilms tumor, immunosuppression, and EBV infection [2]. Worthy of note in this setting is the underestimated diagnosis of malignancy in the excluded stomach because of the difficult endoscopic evaluation of this special anatomical region [8]. Concordant with literature, the patient was in the sixth decade of life, had an insidious clinical course with no-specific abdominal pain, and presented with liver metastasis at diagnosis. And his poor outcome included recent implants in bones, lungs, and the central nervous system. There was no remarkable response of distant metastases to the chemotherapy schedules [6]. Because of the development of brain implants, he was referred to treatment by radiotherapy. Although the results of this therapy are usually poor, that is also a resource utilized in LMS. The first hypothesis of GIST was ruled out by CD117 (c-kit) and CD34 negativity, while the positivity of vimentin V9 and actin 1A4 were consistent with the diagnosis of gastric LMS. Except for the previous RYGB, there was no significant risk factor for gastric malignancy [8]. Late diagnosis and misdiagnosis of gastrointestinal LMS with GISTS are frequent, as well as multiple metastases at diagnosis, unsatisfactory therapeutic responses, and poor outcomes. Cheng et al. reported a 43-year-old woman with a previous resection by Billroth I procedure of a gastric tumor $(3 \mathrm{~cm}) 1$ year ago [1]. Symptoms were weakness and abdominal discomfort. The initial diagnosis was a spindle cell tumor and she had no postoperative adjuvant therapy. Histological, immunohistochemical and genetic evaluations done by authors showed an LMS. The results were SMA $(+)$, calponin $(+)$, CD34 (-); CD117 (-), DOG (-), desmin (doubtful), S-100 (-), Ki-67 $(15 \%+)$; and no KIT exon $9,11,13,17$, or PDGFRA exon 12 or 18 mutation. The patient claimed of low back pain, had severe anemia, liver, and lymph node metastases. She underwent transarterial chemoembolization with oxaliplatin and tetrahydropalmatine emulsion with lipiodol, plus high-intensity focused ultrasound for retroperitoneal metastases. The choice of this alternative management was due to her fragile clinical general status [1].

Gubatan and Shah reported a 50-year-old man with the antecedent of Wilms tumor and presenting with gastrointestinal bleeding by a percutaneous endoscopic gastrostomy tube [2]. The endoscopy showed a large, ulcerated mass on the gastric body. The immunohistochemical study was consistent with LMS [positive for cytokeratin, calponin, actin, and desmin (focal); and negative for CD117, S100, CK5/6, Cam5.2, ALK-1, CD34, CD31, and myogenin]. The patient and his family decided against any kind of therapy because of his poor prognosis [2]. Hasnaoui et al. described the case of a 63-year-old woman, with longstanding anemia due to digestive bleeding and the endoscopy revealed an ulcerated tumor $(9 \mathrm{~cm})$ on the cardia [3]. The immunohistochemistry evaluation revealed positivity for vimentin, smooth muscle actin (SMA), and h-caldesmon, while the immunoreactivity for both KIT and DOG1 was doubtful. With no peritoneal or liver metastasis, she had total gastrectomy with uneventful course [3]. Kang et al. reported a 57-year-old woman with digestive bleeding of long duration caused by a gastric tumor without evidence of metastasis and was resected, with no adjuvant treatment. Immunohistochemical study showed SMA (+), desmin (+), CD117 (-), DOG1 (-), CD34 (-), S-100(-), and Ki-67 index (50\%). For a definitive diagnosis, we performed targeted next-generation sequencing. The genes revealed in the panel included KIT, PDGFRA, SDHA, SDHB, SDHC, SDHD, BRAF, KRAS, NRAS, and EGFR, without mutations in any of them. The patient died with gastrointestinal obstruction and malnutrition one year after surgery [4].

Mehta et al. described a 47-year-old male two years after the diagnosis of a huge gastric LMS $(130 \mathrm{~mm} \times 130 \mathrm{~mm}$ $x 100 \mathrm{~mm}$ ) in the greater curvature, and then excised by laparotomy [6]. Immunohistochemistry studies of primary and liver metastatic lesions showed positivity for alpha-smooth muscle actin, and h-caldesmon, and negativity for CD117, DOG-1, and S100. Two years after receiving six cycles of doxorubicin, multiple liver metastases developed, and he underwent gemcitabinedocetaxel chemotherapy without significant response. Further pazopanib administration resulted in a reduction (approximately 17\%) of metastasis diameter. The authors commented that resection plus chemotherapy is the first option for liver metastases of LMS, but the presence of multiple lesions made the patient not suitable for hepatic resection [6].

\section{Conclusion:}

The evolution of our patient corresponds to descriptions of other cases of this malignancy. The diagnosis of gastric LMS often occurs at late stages because of the insidious clinical course. The rate of liver metastasis at diagnosis is high. Besides, the relatively poor response to the alternative management for non-surgical stages of the disease yields severe outcomes. Close control of the excluded stomach is necessary after RYGB due to the risk of malignancy.

\section{Acknowledgements}

None

\section{Contributors}

NAS, LAMDS, VMDS conceptualized and designed the study, acquired, and analyzed data, interpreted the study results, drafted the manuscript, and critically revised the final version of the manuscript.

\section{Funding}

No funding was received for this study.

\section{Competing interests}

No benefits in any form have been received or will be received from a commercial party related directly or indirectly to the subject of this article.

\section{Availability of data and materials}

Further information is available from the corresponding author on reasonable request.

Ethics approval

Not applicable. 
Provenance and peer review

Not commissioned; externally peer reviewed.

\section{Open access}

This is an Open Access article distributed in accordance with the Creative Commons Attribution NonCommercial (CC BY-NC 4.0) license, which permits others to distribute, remix, adapt, build upon this work noncommercially, and license their derivative works on different terms, provided

the original work is properly cited and the use is non-commercial. See: http://creativecommons.org/ licenses/by-nc/4.0/

\section{References}

[1] Cheng CS, Chen L, Xie J, Chen Z. Multimodality palliative treatment with transarterial chemoembolization and high-intensity focused ultrasound for gastric leiomyosarcoma multiple liver metastasis pain: A case report. Medicine (Baltimore). 2019;98(39):e17328.

[2] Gubatan J, Shah N. Gastric leiomyosarcoma unmasked by bleeding from a percutaneous endoscopic gastrostomy tube. ACG Case Rep J. 2020;7(1):e00301.

[3] Hasnaoui A, Jouini R, Haddad D, Zaafouri H, Bouhafa A, Ben Maamer A, et al. Gastric leiomyosarcoma and diagnostic pitfalls: a case report. BMC Surg. 2018;18(1):62.

[4] Kang WZ, Xue LY, Tian YT. Leiomyosarcoma of the stomach: A case report. World J Clin Cases. 2019;7(21):3575-82.

[5] Kawaguchi K, Igarashi K, Murakami T, Kiyuna T, Nelson SD, Dry $\mathrm{SM}$, et al. Combination of gemcitabine and docetaxel regresses both gastric leiomyosarcoma proliferation and invasion in an imageable patient-derived orthotopic xenograft (iPDOX) model. Cell Cycle. 2017;16(11):1063-9.

[6] Mehta V, Rajawat M, Rastogi S, Phulware RH, Mezencev R. Leiomyosarcoma of the stomach with metastasis to the liver: a case report with review of the literature. Future Sci OA.;4(2):FSO264.

[7] Rastogi S, Kalra K, Manasa P, Rajawat M, Mehta V. Long lasting response of trabectedin in patient with gastric leiomyosarcoma with liver metastasis: an update to previous report. Future Sci OA. 2019;6(1):FSO432.

[8] Ravacci GR, Ishida R, Torrinhas RS, Sala P, Machado NM, Fonseca DC, et al. Potential premalignant status of gastric portion excluded after Roux en-Y gastric bypass in obese women: A pilot study. Sci Rep. 2019;9(1):5582. 